\title{
First Light Detection of A Single Solar Radio Burst Type III Due To Solar Flare Event
}

\author{
Z. S. Hamidi ${ }^{1, *}$, N. N. M. Shariff ${ }^{2}$, C. Monstein ${ }^{3}$ \\ ${ }^{1}$ School of Physics and Material Sciences, Faculty of Sciences, MARA University of Technology, \\ 40450, Shah Alam, Selangor, Malaysia \\ ${ }^{2}$ Academy of Contemporary Islamic Studies (ACIS), MARA University of Technology, \\ 40450, Shah Alam, Selangor, Malaysia \\ ${ }^{3}$ Institute of Astronomy, Wolfgang-Pauli-Strasse 27, Building HIT, Floor J, \\ $\mathrm{CH}-8093$ Zurich, Switzerland \\ *E-mail address: zetysh@salam.uitm.edu.my
}

\begin{abstract}
The eruption mechanism of solar flares and type III are currently an extremely active area of research, especially during the solar cycle is towards maximum. In this case, the total energy of solar burst type III is of the order of solar flare with the explosion of the energy can up to $10^{15}$ ergs. The solar flare event is one of the most spectacular explosions that still be on-going study in the solar physics world. This event occurred at 2:000 UT on $15^{\text {th }}$ April 2012 is due to the explosion of the magnetic energy in from the chromosphere and converted into the heating, mass motion and particle acceleration which can be detected by solar radio burst type III. In this work, we will highlight our first light detection of very tiny solar radio burst type III, which has been observed at the National Space Centre, Banting, Selangor detected by the Compound Low Cost Low Frequency Transportable Observatories (CALLISTO) system at 5:53:23. The region of the data is from $150-400 \mathrm{MHz}$ in radio region. This burst is drifted from $150 \mathrm{MHz}$ till $260 \mathrm{MHz}$. It represents a total energy of $6.2035 \times 10^{-7}$ $\mathrm{eV}-1.0753 \times 10^{-6} \mathrm{eV}$. This fast drift burst is a continuity of the acceleration of the particles which is intermittent, and can be observed since the explosion of the solar flare. Although the burst is very tiny, it is still significant because this burst is the first detection of a single type III burst from our site. Still, the acceleration of the particles can be detected from Earth in the radio region within 3 hours period of observation at the post stage of solar flare.
\end{abstract}

Keywords: Solar flare; low frequency; radio wavelength; solar burst, type III; CALLISTO

\section{INTRODUCTION}

Solar flare is considered as a high energy and complicated phenomenon in which mass eruptions occur, energetic particles are generated and highly energy radiations are emitted. During a flares, large quantities of energy are transferred between the corona and chromosphere through thermal conduction, non-thermal particle beams, radiation transport and mass motions. Indirectly the major particles, so-called the plasma are heated to tens of millions of Kelvins the electrons, protons, and heavier ions are accelerated to near the speed of light. This event is triggered by fast drift of individual sunspot proper motion within the 
complex magnetic configuration due to instabilities of equilibrium of coronal magnetic field [1]. Since the magnetic force is much stronger than other forces in the corona, any coronal structure is mainly controlled by magnetic field.

Based on intensive study, some flares exhibit as 'explosive phase' during the maximum cycle which is associated with hydrodynamic effects. In this scenario, an optical continuum reaches a peak increase of several percent, which is in solar white-light flares based on radiative hydrodynamics models. The previous data showed two different kind of spike event; (1) spike clusters which originating in post-flare phase [2] and (2) originate from the main flare phase and well originate with non-thermal flare emission [3]. It is widely accepted that the solar flare is related to the relatively strongest magnetic field. Previous studies found that the variability under magnetic conditions in electron density, $\mathrm{N}_{e}$, ion temperature $T_{i}$ and electron temperature $T_{e}$, respectively changed with local time and height due to day to day flares monitoring [4]. The eruption mechanism of solar flares and type III are currently an extremely active area of research, especially during the solar cycle is towards maximum cycle. There is a particular focus on their physical properties, as opposed to the microphysics such as that needed for the evolution and formation of this burst or particle acceleration as such. The related theory, model, significant parameters and the behavior of the burst in low range frequency has been discussed in a previous work [5]. In this case, the total energy of solar burst type III is of the order of $10^{15}$ ergs. It occurs in the impulsive phase, which is more intense at meter wavelengths and may have a continuum attached to it [6]. The solar radio burst type III consequences from the ejection of plasma oscillations localized disturbance due to excited in the plasma frequency incoherent radiations such as gyro synchrotron and freefree emissions appear in the radio wavelengths plays a dominant role at the meter and decimeter wavelengths, which may be associated with the flare primary energy-releasing sites [7]. This burst usually associated with a single flare or flare-like events that excited through the lower solar corona in a bunch of electrons which emit $l$-waves (electron plasma waves) incoherently [8]. The electron is thought to be accelerated within a second or so [9]. In between of the process, the instability is formed and sustained through velocity dispersion, whereby the high energy electron fluxes race ahead of the low energy electron fluxes creating a transient bump-on-tail instability in the reduced, one-dimensional distribution $\left(\mathrm{f}_{\mathrm{r}} / \mathrm{v}>0\right)$. In specific range, the event starts with a group of metric type III bursts at $(200-400) \mathrm{MHz}[10]$.

The purpose of this work is to study solar burst type III that leads the understanding of the relationship with solar flare events. We attempt to analysed our data obtained by using the Compound Low Cost Low Frequency Transportable Observatories (CALLISTO) spectrometer.

\section{EXPERIMENTAL}

Solar radio burst research in Malaysia has been started since 2011 by collaborating with an e - CALLISTO network and become one of the most important research in astrophysics research [11]. This is a very good start for developing a radio astronomy in Malaysia [12]. The CALLISTO system consists of the Log Periodic Dipole Antenna (LPDA), a preamplifier, and the spectrometer, which operating from $45 \mathrm{MHz}$ to $870 \mathrm{MHz}$ setup it at the National Space Centre, Banting, Selangor since $20^{\text {nd }}$ February 2012. In this region, we also possible to monitor a different solar type of radio bursts $[13,14]$. It is located at $\left(\mathrm{N} 02^{\circ} 49.488^{\prime} \mathrm{E} 101^{\circ}\right.$ $36.168^{\prime}$ ) where this site is mainly purpose for development of space and radio monitoring 
$[15,16]$. Our first task is by constructing the Log Periodic Dipole Antenna (LPDA) did some testing, calibration process and applied the antenna for solar burst monitoring $[17,18,19]$.

The antenna has a boom length 5.45 meters with gain $7 \mathrm{dBi}$ [15]. The CALLISTO (Compound Astronomical Low-cost Low-frequency Instrument for Spectroscopy in Transportable Observatory) spectrometer, designed and built by electronics engineer Christian Monstein of the Institute for Astronomy of the Swiss Federal Institute of Technology Zurich (ETH Zurich), record the intensity of electromagnetic radiation at radio frequencies, from 45 $-870 \mathrm{MHz}[20]$.

Physically, the system is characterized by the wide range of frequency which can occupy the solar burst [21]. At our site, we could possible to monitor the solar activity within 12 hours throughout a year [22]. Figure 1 shows a very tiny solar radio burst type III. This tiny solar burst is due to the instabilities of the active region where the minor eruption still ongoing in the particular active region. This burst is drifted from $150 \mathrm{MHz}$ till $260 \mathrm{MHz}$. It represents a total energy of $6.2035 \times 10^{-7} \mathrm{eV}-1.0753 \times 10^{-6} \mathrm{eV}$. Still, it is not clear how the magnetic energy is transformed into the particle kinetic energy, nor is it known how the particles are accelerated to energies as high as $10 \mathrm{MeV}$.

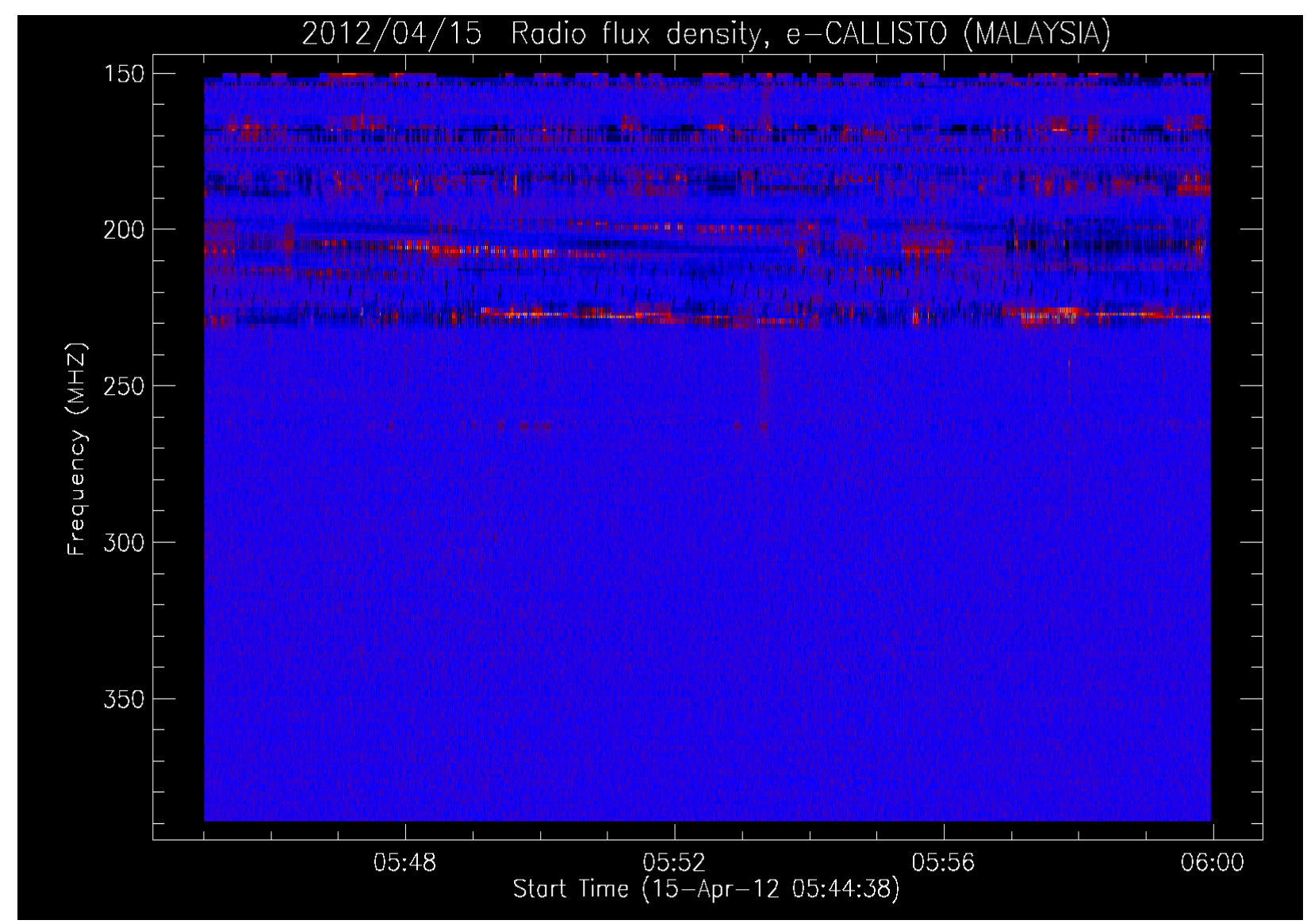

Figure 1. Solar Radio Burst Type III detected at the National Space Centre, Banting Selangor at 5:53:23 UT ON $15^{\text {th }}$ April 2012.

The range of wavelengths of the burst is from 1.9986 meter to 1.1530 meter. This means that the explosion of solar flares is from the corona $\sim 1 \mathrm{R} \odot$. The eruption also might 
because of the inter-correlation of the particle interaction with the other sunspots. The mechanism of the flare is different of each active region.

There are several parameters that need to be considered such as the sunspot number, radio flux and the density of the protons. It should be noted that the is a permanent Radio Frequency Interference that can be observed very clearly in the region from $150-230 \mathrm{MHz}$. This interference is due to an aeronautical mobile-satellite signal. Detailed parameters that relate to this solar event is highlighted in Table 1.

Table 1. Parameters that associated with solar flare on 15 April 2012 (Credicted to Space Weather website).

\begin{tabular}{|c|c|}
\hline Parameter & Value \\
\hline Solar wind speed & $400.8 \mathrm{~km} / \mathrm{sec}$ \\
\hline Density & $0.0 \mathrm{protons} / \mathrm{cm}^{3}$ \\
\hline 6-hr max (Solar Flare Classification) & $\mathrm{B} 3$ \\
\hline 24-hr max (Solar Flare Classification) & $\mathrm{C} 2$ \\
\hline Sunspot number & 65 \\
\hline $10.7 \mathrm{~cm}$ flux & $98 \mathrm{sfu}$ \\
\hline
\end{tabular}

\section{OBSERVATION OF SOLAR FLARE EVENT}

The flare data are mainly taken from the National Oceanic and Atmospheric Administration (NOAA) website. Flares occur in active regions AR1458, where intense magnetic fields penetrate the photosphere to link the corona to the solar interior. It should be noted that the event is exploded at 2:00UT. Still the acceleration of the particles can be detected from Earth in the radio region within 3 hours period of observation.

The illustrations of the solar flare are given in Figure 2 from the Solar Dynamics Observatory (SDO). This image is in ultraviolet region, which it is very clear that the Sun is in active phase with a very strong and large acceleration of particles could be observed from the active region AR1458.

We continue to investigate the effect of the magnetic of the Earth due to the particles acceleration of this event. For this reason, we used a data from Helioseismic and Magnetic Imager (HMI).

Figure 3 shown the image taken from the HMI of the active region 1429. It was found that the probabilities for significant disturbances in Earth's magnetic field is about $10 \%$ at mid altitude and $15 \%$ at high altitude. With this value, there will no explosion of the Coronal Mass Ejections (CMEs) event during this period. This active region also can be observed on $15^{\text {th }}$ April 2012. 


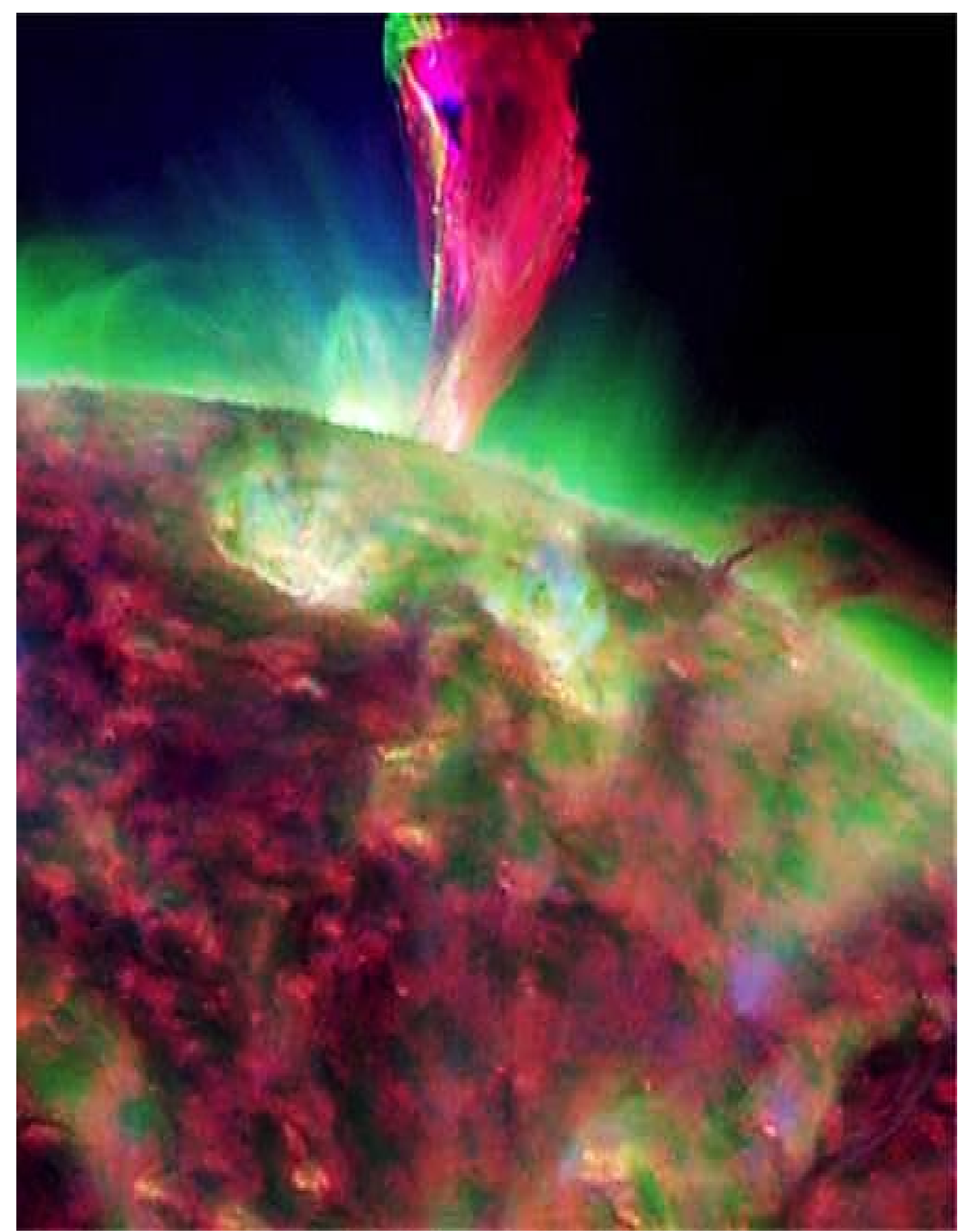

Figure 2. The extreme solar flare in ultraviolet (UV) image ( Credited to: NASA's Solar Dynamics Observatory) 


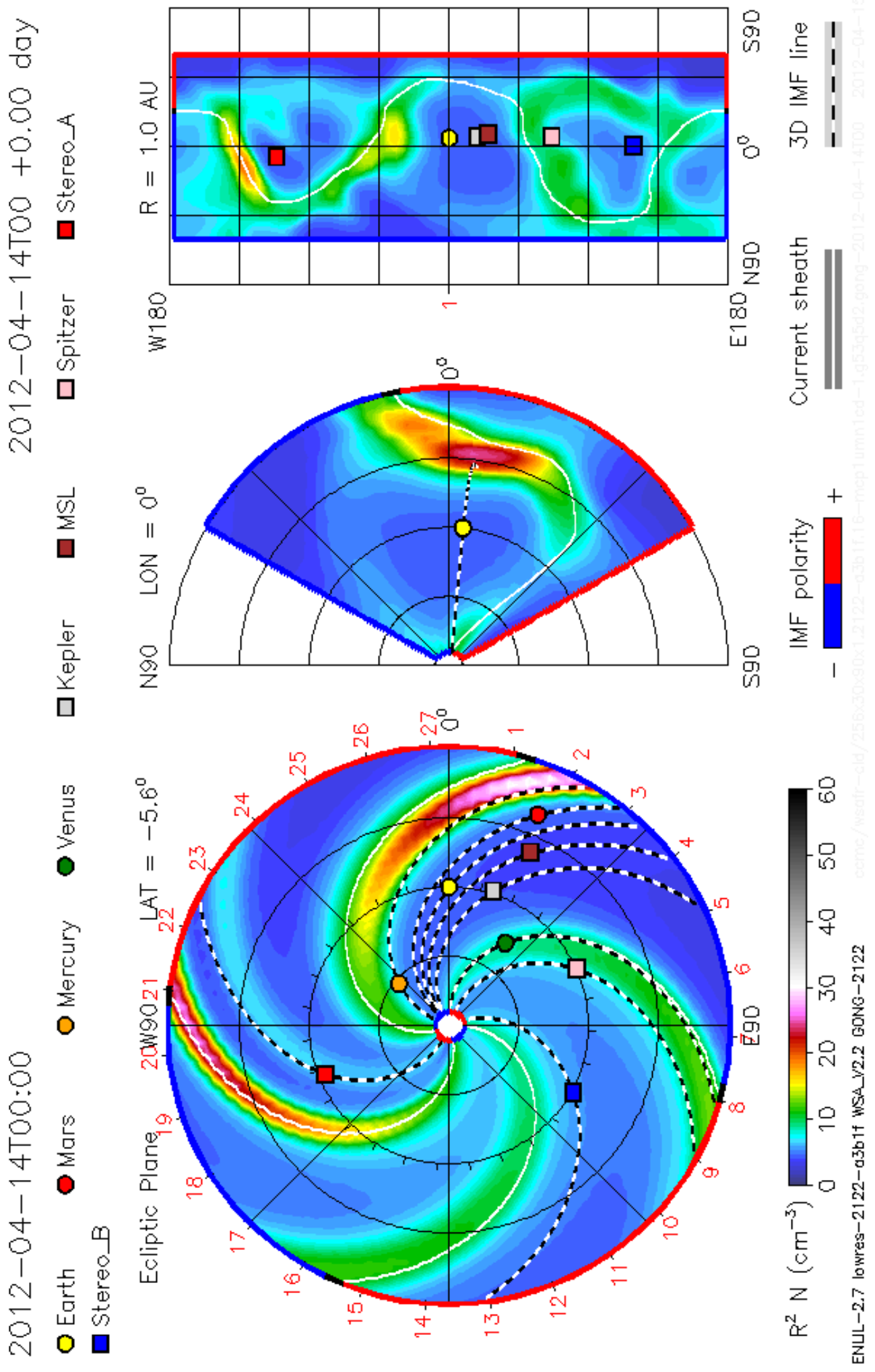

Figure 3. Image from the Helioseismic and Magnetic Imager (HMI) on the Solar Dynamics Observatory (SDO) shows active region 1429 in the upper left corner. The right figure is the enlarge image of AR1429. Credit to NASA/SDO/HMI. 


\section{CONCLUSION}

This event has shown that the solar radio burst type III is very dominant in the meter region in radio wavelengths. This tiny solar burst is an indicator of the post-stage of the eruption of solar flare after the 3 hours period of explosion.

\section{ACKNOWLEDGEMENT}

We are grateful to the LASCO,SDO/AIA, NOAA, Helioseismic and Magnetic Imager (HMI) and SWPC make their data available online. This work was partially supported by the FRGS (600 RMI/FRGS 5/3 2012) UiTM grants. Special thanks to the National Space Agency and the National Space Centre for giving us a site to set up this project and support this project. Solar burst monitoring is a project of cooperation between the Institute of Astronomy, ETH Zurich, and FHNW Windisch, Switzerland, MARA University of Technology and University of Malaya. This paper also used NOAA Space Weather Prediction Centre (SWPC) for the sunspot, radio flux and solar flare data for comparison purpose. The research has made use of the National Space Centre Facility and a part of an initiative of the International Space Weather Initiative (ISWI) program.

\section{BIOGRAPHY}

Dr. Zety Sharizat Hamidi is currently a lecturer and focused in Solar Astrophysics research specifically in radio astrophysics at the School of Physics and Material Sciences, Faculty of Sciences, MARA University of Technology, 40450, Shah Alam, Selangor, Malaysia. Involve a project under the International Space Weather Initiative (ISWI) and also a lecturer in School of Physics and Material Science, at MARA University of Technology, Shah Alam Selangor.

Dr. Nur Nafhatun Md Shariff is a senior lecturer at Academy of Contemporary Islamic Studies (ACIS), MARA University of Technology, 40450, Shah Alam, Selangor, MalaysiaHer current research is more on sustainability; environmental aspect. She is looking forward for cross-field research, i.e. solar astrophysics, light pollution measurement (mapping) and religious studies.

C. Monstein is a senior Engineer at Institute of Astronomy, Wolfgang-Pauli-Strasse 27, Building HIT, Floor J, CH-8093 Zurich, Switzerland and one of the researchers who initiated the CALLISTO system around the world.

\section{References}

[1] D. Herdiwijaya, S. Imelda, Jurnal Matematika dan Sains 11 (2006) 7.

[2] A. O. Benz, Plasma Astrophysics, Springer, Dordrecht, 2002.

[3] M. J. Aschwanden, Güdel M., ApJ 401 (1992) 736-753.

[4] G. D. Fleishman, Gary D. E., Nita G. M., ApJ 593 (2003).

[5] Z. S.Hamidi, U. F. S. U. Ibrahim, Z. Z. Abidin, Z. A. Ibrahim, N. N. M. Shariff, International Journal of Fundamental Physical Sciences 3 (2013) 20-23.

[6] M. V. Goldman, D. F. Smith, Physics of the Sun, Dordrecht: Reidel, 1986.

[7] M. Karlický, Kosugi T., $A \&$ \& 419 (2004).

[8] D. B. Melrose, Australian J. Phys. 23 (1970) 885-903.

[9] P. A. Sturrock, Phys. Rev. Lett. 16 (1966).

[10] A. O. Benz, C. Monstein, A. H. Meyer, Solar Phys. 226 (2005) 143-151.

[11] Z. S. Hamidi, Z. Abidin, Z. Ibrahim, C. Monstein, N. Shariff, International Journal of 
Fundamental Physical Sciences 2 (2012) 32-34.

[12] Z. S. Hamidi, N. N. M. Shariff, C. Monstein, Z.A. Ibrahim, International Letters of Chemistry, Physics and Astronomy 7 (2014) 37-44.

[13] Z. S. Hamidi, N. N. M. Shariff, International Letters of Chemistry, Physics and Astronomy 4 (2014) 29-36.

[14] Z. S. Hamidi, N. N. M. Shariff, Thermal Energy and Power Engineering 3 (2014) 181-184.

[15] Z. S. Hamidi, S. Chumiran, A. Mohamad, N. Shariff, Z. Ibrahim, N. Radzin, N. Hamzan, N. Anim, A. Alias, American Journal of Modern Physics 2 (2013) 4.

[16] Z. S. Hamidi, N. N. M. Shariff, Z. A. Ibrahim, Z. Z. Abidin, Solar Studies in Radio Emission and Optical Photometry, Dimensi Penyelidikan Astronomi Islam, University of Malaya Publisher, University of Malaya, 2013, pp. 33-40.

[17] Z. S. Hamidi, Z. Ibrahim, Z. Abidin, M. Maulud, N. Radzin, N. Hamzan, N. Anim, N. Shariff, International Journal of Applied Physics and Mathematics 2 (2011) 3.

[18] Z. S.Hamidi, N. N. M. Shariff, Evaluation of Signal to Noise Ratio (SNR) of Log Periodic Dipole Antenna (LPDA) Business Engineering and Industrial Applications Colloquium 2013, IEEE, Langkawi, Malaysia, 2013, pp. 434-438.

[19] Z. S. Hamidi, et al., International Journal of Fundamental Physical Sciences 2 (2012) 4.

[20] A. O. Benz, C. Monstein, H. Meyers, CALLISTO, A New Concept for Solar Radio Spectrometer, Kluwer Academic Publishers, The Netherland, 2004.

[21] Z. S. Hamidi, N. Anim, N. N. M. Shariff, Z. Z. Abidin, Z. A. Ibrahim, C. Monstein, Dynamical structure of solar radio burst type III as evidence of energy of solar flares, in: R.Shukor (Ed.), PERFIK 2012, American Institute of Physics, Malaysia, 2013, pp. 11-15.

[22] Z. S. Hamidi, N. Shariff, Z. Abidin, Z. Ibrahim, C. Monstein, Middle-East Journal of Scientific Research 12 (2012) 6. 